\title{
Fixed price and quality signals
}

\author{
Luis C. Corchon* \\ Departamento de Fundamentos del Analisis Economico and Instituto Valenciano de Investigaciones \\ Económicas, Facultad de Ciencias Economicas Universidad de Alicante, San Vicente del Raspeig, \\ Alicante, Spain
}

\begin{abstract}
In this paper I study the existence and efficiency of equilibrium for a fixed-price economy in which the quality of goods is variable.
\end{abstract}

Key words: Equilibrium; Rationing; Fixed price; Efficiency.

\section{Introduction}

One of the most notable achievements of Fixed Price Theory (see Benassy, 1975; Drèze, 1975; Younès, 1975; Barro and Grossman, 1976; and Malinvaud, 1977) has been to show the existence of quantity signals under whose guidance agents can attain compatible decisions. The underlaying message of this literature is that prices are not the unique relevant signal generated by the economic environment. In this paper I will use a signal different from prices or quantity constraints in order to achieve market balance.

The main idea of my paper is that in markets in which prices are rigid, qualities will take their balancing role (see Sheshinski, 1976, for the case of a regulated monopoly that faces parametric prices but can manipulate the quality of the product and Tirole, 1988, p. 287, for an example of oligopolistic firms competing in qualities with fixed prices). The empirical importance of this adjustment remains to be seen, but casual empiricism suggests that it might be significant, especially in planned economies in which the demands of the Central Authority are sometimes fulfilled, altering the size, weight, etc. of goods. Apparently this is one of the reasons for the introduction of 'market-oriented' incentives. As will be seen, our results imply that the market does not necessarily solve these problems.

${ }^{*}$ I wish to acknowledge P. Chander, X. Freixas, O. Hart, C. Herrero, A. Kirman, J.-J. Laffont, L. Makowski, J. Silvestre, J.A. Trujillo, A. Villar and two anonymous referees for deep comments and suggestions. All errors are my own. I also thank D.G.I.C.Y.T. under projects PB88-0289 and PB91-0756 for financial support. This paper is dedicated to Frank H. Hahn. 
In this paper it is shown that in a fixed-price economy in which the quality of goods can be changed, a market equilibrium with no rationing exists. As was noted above, the idea behind our notion of equilibrium is that under fixed prices a (notional) excesses demand of a good creates a negative pressure on the quality of this good. Similarly, excess supply gives incentives to enhance quality. Examples such as hospitals, food, universities, public transport, restaurants, furniture, coffee, etc. come easily into one's mind. ${ }^{1}$

In this paper firms and consumers are assumed to be 'quality-takers', i.e. they maximize their respective objective functions object to the usual constraints and taking qualities as given. This means that I do not model how qualities are set by agents. Instead, I stick to a much more elusive procedure. I simply assume that when there is some discrepancy between supply and demand, 'the market' changes the quality of goods in order to match them. ${ }^{2}$ In this way, quality standards in my model mimic the role played by prices in the Arrow-DebreuMcKenzie model or by quantity constraints in the standard fixed price literature. Therefore my model is successful in determining quality standards at the cost of leaving price levels completely unexplained.

I show that under standard continuity and convexity conditions plus an additional assumption which ensures that utility functions and production sets are actually affected by the quality levels, there is an allocation in which all agents maximize and every market balances. I call this allocation a quality-taking equilibrium. The formal proof of the existence of such an allocation is recorded as Proposition 1.

Two remarks are in order. First, I assume that quality varies continuously, with a range contained in a compact subset of the real line. This is not only a natural approach in many cases but it is also convenient since the continuity of the signal is an almost necessary requirement in order to achieve market balance. Secondly, I assume that quality levels are common knowledge for all agents in the economy and therefore many interesting problems associated with qualities and asymmetrical information are entirely disregarded (see, for example, the pioneering paper by Akerlof, 1970).

The following step is to study the efficiency of this kind of equilibrium. It is easy to show that even though first-order conditions of Pareto efficiency and market equilibrium will generally differ, the allocation of goods is Pareto efficient given quality standards (see Proposition 2). This follows from the fact that, for a given quality level, my equilibrium is an Arrow-Debreu-McKenzie (perfectly competitive) equilibrium in which all agents maximize their objective functions taking prices as given and all markets clear. Moreover, under additional assumptions I show that the following possibilities may arise.

\footnotetext{
${ }^{1}$ One possible motivation for my approach is that, under price inflexibility, firms do not profit from rationing so, if feasible, they would prefer to change qualities. This is analogous to the result obtained by Bohm et al. (1983) on the lack of incentive of a monopoly to establish quantity constraints.

${ }^{2}$ The foundation of this assumption by means of game-theoretical models must be the subject of further research.
} 
(1) A quality-taking equilibrium can dominate (in the Pareto sense) other quality-taking equilibria even if there is a strong agreement of preferences on the quality level. We recall that the fact that some equilibria may dominate, in the Pareto sense, other equilibria occurs in other second-best problems (for instance, see Hart, 1975, in the context of an economy with missing markets).

(2) If for some economy the quality-taking allocation is Pareto efficient, this economy is 'exceptional' in the set of economies, i.e. the economy with a Pareto-efficient quality-taking equilibria is 'surrounded' by economies whose quality-taking equilibria are not Pareto efficient.

Points (1) and (2) above are formally recorded as Propositions 3 and 4 . It should be noted that this kind of inefficiency is of a different sort from that studied by Nayak (1980) and Silvestre (1985) in the framework of a fixed-price economy with rationing, but it does bear some similarity with problems considered by Hart (1980) and Makowski (1980) in large, purely competitive economies.

Finally, the rest of the paper is organized as follows. In Section 2 I explain the model and the main concepts, including that of a quality-taking equilibrium. In Section 3 I prove the existence of such an equilibrium. Section 4 is devoted to the study of the welfare properties of this equilibrium. The final section comments on several implications of my approach and offers some suggestions for future research.

\section{The model}

There are $n$ goods, $h$ consumers and $f$ firms $(n, h$ and $f$ are assumed to be natural numbers). Let $H=\{1 \ldots h\}, N=\{1 \ldots n\}$ and $F=\{1 \ldots f\}$ be the sets of consumers, goods and firms, respectively. Let $j, i$ and $g$ be typical elements of $H, N$ and $F$, respectively. Consumer $j$ 's initial endowments and shares are denoted, respectively, by non-negative vectors $\bar{x}_{j}=\left(\bar{x}_{j 1} \ldots \bar{x}_{j n}\right)$ and $\theta_{j}=\left(\theta_{j 1} \ldots \theta_{j f}\right)$ such that shares of any firm add up to one. Let $k_{i}$ be the quality of good $i$ where $k_{i} \in\left[0, \bar{k}_{i}\right] \equiv K_{i}, \bar{k}_{i} \neq 0, \forall i \in N$.

Let $K=\times_{i=1}^{n} K_{l}$ be the quality space with typical element $k=\left(k_{1} \ldots k_{n}\right)$. Each consumer, say $j$, is endowed with a utility function $U_{j}: \mathfrak{X}_{j} \times K \rightarrow \mathbb{R}$, where $\mathfrak{X}_{j} \subseteq \mathbb{R}_{+}^{n}$ is $j$ 's consumption set. Each firm, say $g$, is endowed with a production correspondence $\tilde{Y}_{g}: K \rightarrow \mathbb{R}^{n}$, where $\bar{Y}_{g}(k)$ is a non-empty subset of $\mathbb{R}^{n}$ which describes the technologically feasible input-output vectors given $k$ (inputs are negative numbers, outputs are positive). Prices, denoted by $\left(\bar{p}_{1} \ldots \bar{p}_{n}\right)$ are assumed to be strictly positive. An economy $e$ is a tuple

$$
\begin{aligned}
e=\left(\bar{p}_{i}, \mathfrak{X}_{j}, U_{j}, \bar{x}_{j}, \theta_{j}, \bar{Y}_{g}, K_{i}\right), \quad i=1 \ldots n, j=1 \ldots h, \\
g=1 \ldots f .
\end{aligned}
$$

Notice that the differences with the standard definition of an economy are that: 
(a) utility functions and production sets depend on quality levels. In particular, notice that there is a production set for every vector in $K$ and (b) prices are part of the environment since they are exogenously given.

Also notice that it is implicit in our approach that $\bar{x}_{j}$ can be freely converted into initial endowments of any quality. If any input is needed for such a conversion, we will say that this agent is a producer, i.e. she has access to a production set which transforms a part of her initial endowments into a good with the required quality. A different approach to the same problem would consist in taking $\bar{x}_{j}$ as being dependent on $k$. This can be accomplished in our model with straightforward modifications.

Definition 1. A quality-taking equilibrium for the above economy is a tuple $\left(x_{1}^{*} \ldots x_{h}^{*}\right)\left(y_{1}^{*} \ldots y_{f}^{*}\right)\left(k_{1}^{*} \ldots k_{n}^{*}\right)$ such that $\forall i \in N, \forall j \in H, \forall g \in F$ :

(a) $k_{i}^{*} \in K_{i}$

(b) $Z_{i}^{*} \equiv \Sigma_{j \in H} x_{j i}^{*}-\Sigma_{g \in F} y_{g i}^{*}-\Sigma_{j \in H} \bar{x}_{j i}=0$;

(c) $x_{j}^{*}$ maximizes $U_{j}\left(x_{j}, k^{*}\right)$ on $B_{j}=\left\{x_{j} \in \mathfrak{X}_{j} / \bar{p} \circ x_{j} \leq \bar{p} \circ \bar{x}_{j}+\Sigma_{g \in F} \theta_{j g} \bar{p}^{\circ} y_{g}^{*}\right\}$;

(d) $y_{g}^{*}$ maximizes $\bar{p} \circ y_{g}$ on $y_{g} \in \bar{Y}_{g}\left(k^{*}\right)$.

Notice that Definition 1 is similar to that of a competitive equilibrium since (c) and (d) require the maximization of utility and profits at given prices and qualities and (b) is just the standard feasibility requirement which is assumed to hold with equality. ${ }^{3}$

\section{The existence of a quality-taking equilibrium}

The strategy of the proof is the following. I first prove the existence of a pseudo-equilibrium. In this kind of equilibrium, consumers and producers maximize according to parts (c) and (d) in Definition 1 above, and I introduce a new, fictitious agent - the auctioneer - in order to set up qualities. It will be seen that a pseudo-equilibrium is not necessarily feasible - part (b) of Definition 1-which means that $I$ have to introduce an additional assumption in order to prove feasibility (i.e. that $Z_{i}=0, \forall i=1 \ldots n$ ).

Definition 2. A tuple $\left(x_{j}, y_{g}, k_{i}\right), i=1 \ldots n, g=1 \ldots f, j=1 \ldots h$, is said to be a pseudo-equilibrium if conditions (a), (c) and (d) in Definition 1 are satisfied and in addition:

(e) $\sum_{i=1}^{n} k_{i} \circ Z_{i}$ is a minimum over $K$ for given $\left(Z_{1} \ldots Z_{n}\right)$.

\footnotetext{
${ }^{3} \mathrm{~A}$ problem of the above notion of equilibrium is that the utility of a consumer in a quality-taking equilibrium may be less than the utility obtained by consuming her initial endowments freely converted at the best qualities, i.e. the quality-taking equilibrium is not necessarily individually rational. In order to avoid this, we may partition the commodity space into resources and produced goods and endow consumers with only resources.
} 
Notice that condition (e) attempts to capture the role of quality standards as balancing supply and demand, i.e. goods in excess demand are given zero quality standards and goods in excess supply are given the maximum possible quality. I first give a list of the sufficient assumptions for the existence of a pseudoequilibrium.

Assumption 1. $\mathfrak{X}_{j}=\mathbb{R}_{+}^{n}, \forall j \in H$.

Assumption 2. $U_{j}\left(x_{j}, k\right)$ is continuous in $x_{j}$ and $k$ and quasi-concave in $x_{j}$ for given $k, \forall j \in H$.

Assumption 3. $\exists \tilde{x}_{j} \in \mathfrak{X}_{j}$ such that $\bar{p} \circ \tilde{x}_{j} \leq \bar{p} \circ \bar{x}_{j}, \forall j \in H$.

Assumption 4. If $k_{i}=\bar{k}_{i}$ some $i \in N$, then $U_{j}$ displays no local satiation $\forall j \in H$.

Assumption 5. The correspondence $\bar{Y}_{g}(k)$ is continuous with convex and compact image sets and $0 \in \bar{Y}_{g}(k), \forall k \in K, \forall g \in F$.

All these assumptions are standard except Assumption 4. An interpretation of this assumption is that if good $i$ is supplied at the highest quality, no consumer is satiated by it. Then we have the following:

Lemma 1. Under Assumptions 1-5 a pseudo-equilibrium exists.

Proof. Let $x_{j}=x_{j}\left(k, y_{1} \ldots y_{f}\right)$ and $y_{g}=y_{g}(k)$ be the correspondences whose values maximize $U_{j}$ and $\bar{p} y_{g}$, respectively, over the sets described under (c) and (d) in Definition 1 (notice that $x_{j}(\cdot)$ depends on $\left(y_{1} \ldots y_{f}\right)$ via profits). By the maximum theorem (see Debreu, 1982, p. 701, Lemma 1), $x_{i}(\cdot)$ and $y_{g}(\cdot)$ are closed correspondences. Also, they are convex-valued. Then, the usual argument (see Debreu, 1982, p. 702, Theorem 3) proves the lemma.

A pseudo-equilibrium is not necessarily feasible (part (b) in Definition 1). For instance, if utility functions and production sets do not depend on $k$, a pseudoequilibrium will always exist but a quality-taking equilibrium will exist only in exceptional cases. In order to avoid such a problem I assume the following.

Assumption 6. (a) $\forall i \in N$ if $k_{i}=0$, then $U_{j}$ is strictly decreasing on $x_{j i}, \forall j \in H$. (b) $\forall i \in N$ if $k_{i}=0$, and $y_{g} \in \bar{Y}_{g}(k)$, with $y_{g i}<0$, then $y_{g}^{\prime}$, which is identical to $y_{g}$ except that $y_{g i}^{\prime}=0$, is such that $y_{g}^{\prime} \in \bar{Y}_{g}(k), \forall g \in F$.

Condition (a) says that if the quality of good $i$ is zero, all consumers dislike it, i.e. it is a 'bad'. Condition (b) says that if any firm uses input $i$ at quality $k_{i}=0$, this input is irrelevant for production purposes (presumably because the firm freely - disposes of any quantity of such an input). Thus, under this assumption, 
$k_{i}=0$ might be interpreted as the worst possible quality for good $i$. An important implication of Assumption 6 is that if $k$ contains an element, say $k_{i}$, equal to zero, then $Z_{i}(k)$ is non-positive. As a referee has pointed out, this allows trivially for a free-disposal quality-taking equilibrium in which $k=0$ and $Z \leq 0$. However, if $Z_{i}(k)>0$ at $k_{i}=0$ there are economies fulfilling Assumptions $1-5$ for which a quality-taking equilibrium does not exist. Now I am prepared to prove my main result:

Proposition 1. Under Assumptions 1-6 a quality-taking equilibrium exists.

Proof. In view of Lemma 1 I only have to show that a pseudo-equilibrium is a quality-taking equilibrium, i.e. that the feasibility condition holds. I denote by $Z_{i}^{+}$ (resp. $k_{i}^{+}$) the value of $Z_{i}$ (resp. $k_{i}$ ) in a pseudo-equilibrium. Suppose that $Z_{i}^{+}>0$ some $i \in N$. Then the minimization of $k \circ Z^{+}$yields $k_{i}^{+}=0$, but this implies (by Assumption 6) that $Z_{i}^{+} \leq 0$, which is a contradiction. Therefore $Z_{i}^{+} \leq 0, \forall i \in N$. If $Z_{i}^{+}<0$ some $i, k_{i}^{+}=\bar{k}_{1}$ and Assumption 4 implies that $\bar{p} \circ Z^{+}=0$. But this contradicts $Z_{i}^{+}<0$, since $\bar{p}$ is assumed to be strictly positive. Hence $Z^{+}=0$.

\section{Some welfare implications}

In this section I study the efficiency of the quality-taking equilibrium. First notice that this equilibrium is an Arrow-Debreu-McKenzie perfectly competitive equilibrium for given quality standards. Therefore, inefficiencies must be due to the (wrong) quality levels, since the distribution of goods, given the equilibrium level of qualities, is efficient. This is related to the work of Hart (1980) and Makowski (1980) on the efficiency of Walrasian equilibria with inactive markets. The model studied here can be interpreted as a model in which many markets are not open and the number of potentials goods in infinite.

Proposition 2. Given the vector of qualities in a quality-taking equilibrium, there is no feasible allocation of goods in which all consumers are better off.

Proof. Identical to the first fundamental theorem in Welfare Economics.

Let us now study the full efficiency of quality-taking equilibria. My results here must be understood as extended examples, i.e. as an illustration of certain possibilities. In particular I show that under some assumptions

(a) there is a continuum of quality-taking equilibria and all consumers are better off in one of them (i.e. equilibria can be Pareto-ranked), and

(b) if an economy, say $e$, has a Pareto-efficient quality-taking equilibrium, then there is a continuum of economies around $e$ such that any quality-taking equilibrium is inefficient.

In order to study (a) above I introduce two new assumptions. 
Assumption 7. $\bar{Y}_{g}\left(k^{*}\right)=\bar{Y}_{g}\left(\varepsilon k^{*}\right), \forall g \in F, \varepsilon \simeq 1$.

Assumption 8. $U_{j}\left(x_{j}, k\right)$ in a vicinity of the quality-taking equilibrium is such that (a) $U_{j}\left(x_{j}, k\right)>U_{j}\left(x_{j}, \lambda k\right), \forall \lambda<1, \forall j \in H$ and

(b) preferences over any two bundles are not affected by a change from $k$ to $\varepsilon k$, $\varepsilon \simeq 1, \forall j \in H$.

Notice that Assumption 7 and Assumption 8(b) are assumed to hold locally. Assumption 7 says that production sets are invariant if all quality standards change slightly in the same proportion. Thus if a firm produces corn from labor and corn, and if the quality of both inputs and output changes slightly, the production possibility set remains the same. Sufficient conditions for Assumption 8 to hold are that utility functions are homogeneous of degree one on quality standards and that they are additively separable on quality standards and quantities. Then a small proportional change in all quality levels transforms monotonically the utility function holding the indifference map among goods constant. Hence both assumptions imply that excess demand functions are homogeneous of degree zero on quality standards in a vicinity of the quality-taking equilibrium. Then I am prepared to prove the following result:

Proposition 3. Let us assume Assumptions 1-8. Then there is a continuum of Pareto-inefficient quality-taking equilibria which can be Pareto-ranked.

Proof. Let $\left(x_{i}^{*}, y_{g}^{*}, k_{i}^{*}\right), j \in H, i \in N, g \in F$, be a quality-taking equilibria (which exists because of Assumptions 1-6). Notice that since in equilibrium excess demands are zero, Assumption 6 implies that $k_{i}^{*} \neq 0, \forall i \in N$. Consider the allocation $\left(x_{j}^{*}, y_{g}^{*}, \varepsilon k_{i}^{*}\right), j \in H, i \in N, g \in F$, with $\varepsilon<1$. If $\varepsilon$ is sufficiently close to 1, Assumption 7 and Assumption 8(b) imply that this allocation is also a quality-taking equilibrium which - because of Assumption 8(a) - is Pareto-dominated by $\left(x_{j}^{*}, y_{g}^{*}, k_{i}^{*}\right), j \in H, i \in N, g \in F$. Finally, notice that choosing $\varepsilon$ adequately we can generate an infinite number of such equilibria in a vicinity of any predetermined quality-taking equilibrium.

Proposition 3 has been obtained under rather strong assumptions, but the economic sense behind it is clear. If all quality standards change proportionally downwards, the demand and supply of any good remains constant since the quality of substitutes varies in the same proportion. Thus the welfare of each consumer decreases. In other words, Proposition 3 shows the inability of the market to find the right quality standards in situations in which excess demand depends on relative quality standards, but utility depends on absolute quality standards. The next proposition shows that even if the Pareto-efficient level of quality standards is unique, generic inefficiency is obtained. In order to do that, I first define what I mean by a Pareto-efficient allocation in our framework. 
Definition 3. An allocation $\left(x_{j}, y_{g}, k_{i}\right), j \in H, i \in N, g \in F$, is Pareto efficient if

(a) $k_{i} \in K_{i}, \forall i \in N$

(b) $\Sigma_{j \in H} x_{j i}-\Sigma_{g \in F} y_{g i}-\Sigma_{j \in H} \bar{x}_{j i}=0, \forall i \in N$;

(c) $x_{j} \in \mathfrak{X}_{i}, y_{g} \in \bar{Y}_{g}(k), \forall j \in H, \forall g \in F$;

(d) there is no $\left(x_{j}^{\prime}, y_{g}^{\prime}, k_{i}^{\prime}\right) j \in H, i \in N, g \in F$, fulfilling (a), (b) and (c) above and such that $U_{j}\left(x_{j}^{\prime}, k^{\prime}\right)>U_{j}\left(x_{j}, k\right), \forall j \in H$.

Notice that Definition 3 corresponds to the standard definition of a weakly Pareto-efficient allocation except that quality levels explicitly enter into the picture. Also notice that I only allow one quality for each good to be produced in order to make a fair comparison between what can be achieved by the market and by a well-informed and benevolent planner (it is clear that the planner can improve market allocations in many instances if two different quality standards of the same good could be produced). I now introduce three new assumptions.

Assumption 9. There is a unique consumer with a strictly quasi-concave and continuously differentiable utility function such that if $a \in$ interior $\mathfrak{X}_{j}$ and $c \in$ boundary $\mathfrak{X}_{j}$, then $U_{j}(a, k)>U_{j}(c, k)$ for any strictly positive $k . \mathfrak{X}_{j}=\mathbb{R}_{+}^{n}$

Assumption 10. The vector of Pareto-efficient qualities for an economy $e$, written $k(e)$, is strictly positive and unique.

Assumption 11. $\forall k \gg 0, \quad \exists\left(y_{1} \ldots y_{g}\right) \in \times_{g \in F} Y_{g}(k)$ such that $\sum_{g \in F} y_{g i}-$ $\sum_{j \in H} \bar{x}_{j i} \gg 0$.

A sufficient condition for Assumption 9 to hold is that preferences over goods can be represented by a Cobb-Douglas utility function. Notice that under Assumption 9, Assumption 10 holds generically, i.e. if for some economy there are two vectors of Pareto-efficient quality standards, a small perturbation of the utility function of the consumer will make Assumption 10 true. Finally Assumption 11 is just a productivity assumption. I now specify my space of economies which will be denoted by $\mathscr{E}$. Let prices vary in an $n-1$ dimensional simplex and keep the rest of the characteristics constant. Therefore an economy is simply a price vector and thus $\mathscr{E}$ contains an uncountable number of elements. I assume that Assumptions 1-6 so a quality-taking equilibrium exists for any $e \in \mathscr{E}$. Then, I show the following:

Proposition 4. Under Assumptions 1-6, 9, 10 and 11 there is only one economy in $\mathscr{E}$ such that the quality-taking equilibrium is Pareto efficient.

Proof. First notice that for every $e \in \mathscr{E}$ the Pareto-efficient allocation of goods to the consumer (denoted by $x(e)$ ) is the same. Moreover, this allocation is unique because Pareto-efficient qualities are unique and Assumptions 5 and 9. Also, it follows from Assumptions 9, 10 and 11 that $x(e) \gg 0$. Therefore there is a single 
element in $\mathscr{E}$ (i.e. a unique price vector in the $n-1$ simplex) for which

$$
\frac{\partial U_{j}(x(e), k(e)) / \partial x_{i}}{\partial U_{j}(x(e), k(e)) / \partial x_{r}}=\frac{p_{i}}{p_{r}}, \quad \forall i, r \in N,
$$

which is the necessary and sufficient condition of the consumer's maximization program in a quality-taking equilibrium. Thus the proposition is proved.

Again strong assumptions are used to justify an intuitive proposition: if qualities are used to clear markets, only in exceptional cases they will be optimal.

\section{Conclusions}

In this paper I have studied the existence and the efficiency of a market equilibrium in which prices are fixed and the quality of goods is used in order to clear up markets. My main conclusions are the following.

(1) If excess-demand functions are responsive enough to quality standards and, in particular, if for the lowest level of quality of any good there is an excess supply of this good (Assumption 6), under standard convexity and continuity assumptions a quality-taking equilibrium exists (Proposition 1).

(2) Given equilibrium quality standards, the allocation of goods is such that no other feasible allocation of goods will improve the welfare of all consumers (Proposition 2).

(3) Under additional assumptions we prove the existence of a continuum of equilibria which are Pareto-ranked (Proposition 3), and the generic inefficiency of equilibria (Proposition 4). Therefore, in this case, the wrong set of quality standards is chosen by the market.

A consequence of my approach is that market failure under fixed prices may be of a different sort than that arising from quantity rationing. In order to illustrate this, consider the following example. Take the usual fixed-price model (e.g. see Malinvaud, 1977) and suppose that at some prices there is unemployment (i.e. we are either in the Classical or the Keynesian unemployment region). Now consider that the quality of labor may change and workers may work harder or work extra hours. Then there is a quality level of labor such that at the initial price level, supply equals demand in the labor market. This allocation is Pareto efficient given quality standards, and involves no involuntary unemployment. However, such an outcome may be very inefficient from the welfare point of view. The point is that to distinguish between that which is voluntary (or notional) and that which is involuntary may not be enough to understand all the possible hidden inefficiencies of an economy. In particular, characteristics which may be attributed to preferences (i.e. laziness of workers) may be due to the wrong levels of quality since any rational agent will be lazy under certain circumstances.

Other possible applications of the basic idea of the paper are (1) the introduction of quality-making firms (see Tirole, 1988, Chapter 7, for a review of 
quality-making firms with flexible prices); (2) the study of the relative efficiency of quantity-rationing equilibria vs. quality-taking equilibria; (3) the consideration of price vs. quality changes in a supergame framework; (4) the study of how quality - which might be interpreted here as technical knowledge - changes with time; and (5) a model in which the range of a priori variation of both prices and qualities is exogenously given and such that if this range is not large enough to achieve market balance, rationing occurs (the model presented here is a special case of this model). In particular it will be interesting to see if such an equilibrium exists without Assumption 6. All this is left to future research.

\section{References}

G.A. Akerlof, The market for lemons: Qualitative uncertainty and the market mechanism, Quart. J. Econom. 84 (1970) 488-500.

R.J. Barro and H.I. Grossman, Money, Employment and Inflation (Cambridge University Press, Cambridge, 1976).

J.P. Benassy, Neokeynesian disequilibrium theory in a monetary economy, Rev. Econom. Studies 42 (1975) 503-523.

V. Bohm, E. Maskin, H. Polemarchakis and A. Postlewaite, Monopolistic quantity rationing, Quart. J. Econom., Suppl. (1983) 189-197.

G. Debreu, Existence of competitive equilibrium in: K.J. Arrow and S. Honkapohja, eds., Handbook of Mathematical Economics (North-Holland, Amsterdam, 1982) Chapter 15, pp. 689-742.

J. Drèze, Existence of an exchange equilibrium under price rigidities, Int. Econom. Rev. 16 (1975) 301-320.

O. Hart, On the optimality of equilibrium when the markets are incomplete, J. Econom. Theory 11 (1975) 418-443.

O. Hart, Perfect competition and optimal product differentiation, J. Econom. Theory 22 (1980) 279-312.

L. Makowski, Perfect competition, the profit criterion and the organization of economic activity, J. Econom. Theory 22 (1980) 222-241.

E. Malinvaud, The Theory of Unemployment Reconsidered, Yrjö Joahnsson Lectures (Basil Blackwell, Oxford, 1977).

P.R. Nayak, Efficiency of non-Walrasian equilibria, Econometrica 48 (1980) 127-134.

E. Sheshinski, Price, Quality and quantity regulations in monopoly situation, Economica 43 (1976) 127-137.

J. Silvestre, Voluntary and efficient allocations are Walrasian, Econometrica 53 (1985) 807-816.

J. Tirole, The Theory of Industrial Organization (M.I.T. Press, Cambridge, MA, 1988).

Y. Younès, On the role of money in the process of exchange and the existence of a non-Walrasian equilibrium, Rev. Econom. Studies 42 (1975) 489-501. 